\title{
EFECTOS DEL LIDERAZGO ESCOLAR EN EL APRENDIZAJE
}

Cifuentes-Medina, José Eriberto; González-Pulido, José Weymar; González-Pulido, Alexandra EFECTOS DEL LIDERAZGO ESCOLAR EN EL APRENDIZAJE

PANORAMA, vol. 14, núm. 26, 2020

Politécnico Grancolombiano, Colombia

Disponible en: http://www.redalyc.org/articulo.oa?id=343963784005

DOI: https://doi.org/10.15765/pnrm.v14i26.1482

Esta obra está bajo una Licencia Creative Commons Atribución-NoComercial-SinDerivar 4.0 Internacional. 
PANORAMA, vol. 14, núm. 26, 2020

Politécnico Grancolombiano, Colombia

Recepción: 16 Agosto 2019

Aprobación: 03 Febrero 2020

DOI: https://doi.org/10.15765/ pnrm.v14i26.1482

Redalyc: http://www.redalyc.org/ articulo.oa?id $=343963784005$

\section{Artículos de investigación científica y tecnológica}

\section{EFECTOS DEL LIDERAZGO ESCOLAR EN EL APRENDIZAJE}

\author{
EFFECTS OF LEADING SCHOOL IN LEARNING \\ EFEITOS DA ESCOLA LEADING NA \\ APRENDIZAGEM
}

\author{
José Eriberto Cifuentes-Medina \\ Joseeriberto.cifuentes@uptc.edu.co \\ Universidad Santo Tomás., Colombia \\ José Weymar González-Pulido \\ Joseweymar.gonzalez@uptc.edu.co \\ Universidad Metropolitana de Educación, Ciencia y Tecnología., \\ Colombia \\ Alexandra González-Pulido \\ Alexandra.gonzalez@uptc.edu.co \\ Universidad Nacional Abierta y a Distancia., Colombia
}

Resumen: El liderazgo es una de las variables que, en el contexto educativo, tiene mayor relevancia en los resultados escolares. El presente artículo teórico busca entender la forma como el liderazgo escolar o pedagógico se ha conceptualizado y los modelos causales que explican su correlación con los resultados de aprendizaje de los estudiantes. La metodología utilizada es de revisión documental. Se encuentra que el concepto de liderazgo escolar no lleva más de un siglo de ser analizado, pasando de modelos rígidos, burocráticos y netamente administrativos a modelos flexibles y distribuidos. Existe evidencia teórica y empírica de la relación positiva, directa e indirecta, entre liderazgo y resultados de aprendizaje estudiantil. Se concluye que es más común la relación causal indirecta y recíproca entre liderazgo, variables mediadoras y aprendizaje, pues existe un "efecto derrame", en donde las dimensiones de liderazgo influyen de manera más amplia en las dimensiones de variables mediadoras y moderadoras, y estas últimas influyen en el resultado de los estudiantes.

Palabras clave: Liderazgo escolar, aprendizaje, liderazgo distribuido, eficacia educativa, calidad educativa.

Abstract: Leadership is one of the variables that in the educational context has greater relevance in school results. The present theoretical article seeks to understand the way in which school or pedagogical leadership has been conceptualized and the causal models that explain its correlation with the results of student learning. The methodology used is of documentary review. It is found that the concept of school leadership has not been analyzed for more than a century, going through rigid, bureaucratic and purely administrative models to flexible and distributed models. There is theoretical and empirical evidence of the positive, direct and indirect relationship between leadership and student learning outcomes. It is concluded that the indirect and reciprocal causal relationship between leadership, mediating variables and learning is more common because there is a "spillover effect" in which the dimensions of leadership influence more broadly the dimensions of mediating and moderating variables and these latter variables influence in the result of the students.

Keywords: School leadership, learning, distributed leadership, educational effectiveness, educational quality. 
Resumo: O líder é uma das variáveis que estão no contexto educativo tem mais relevância em estudos escolares. Apresente o ensaio teórico da pesquisa como forma de liderar a escolaridade pedagógica e os modelos causais que explicam a correlação com os resultados de aprendizado dos estudantes. La metodología utilizada es de revisión documental. A encuentra that el concepto de liderar schools the lleva más de un siglo de seralized, pasando por textiles hyper, burocratic and netamente administrative model flexibles y distribuidos. Evidence teórica y empírica of the positive positive, directa and indirecta, between liderazgo and results of learning study. Se concluye que a mais comum é a relação causal indireta e recíproca entre lideranças, variáveis mediadoras e aprendizagens pue existe um "efeito derrame" nas dimensões das lideranças influentes de gênero mais amplas nas dimensões de variáveis mediadoras e moderadoras e estas últimas variáveis influentes en el resultado dos estudiantes.

Palavras-chave: Liderança escolar, aprendizado, liderar distribuído, eficacia educativa, calidad educativa.

\section{INTRODUCCIÓN}

Dentro de las conductas humanas, el liderazgo surge naturalmente y por instinto. En la época primitiva la organización en manadas y el surgimiento de un líder se da por supervivencia, pues el ser humano se siente seguro si está cerca a alguien fuerte, que marque una ruta e inspire a seguirla. Hoy en día no es muy distinto; en la política, en las empresas, en el mundo educativo surgen y se necesitan líderes gracias a que estos mismos instintos permanecen en nuestra biología: nos sentimos más seguros con alguien fuerte que marque el rumbo y que inspire a seguirlo.

El liderazgo se encuentra inmerso en todas las facetas de la vida, en consecuencia, existen tantas definiciones de liderazgo como autores han tratado de conceptualizarlo. Cada uno de ellos, desde su propia praxis, le encuentra significados diferentes. No obstante, todas las definiciones finalmente convergen a una sola: el liderazgo es influencia (Maxwell, 2007, p.1), como se puede constatar en Bhattacharyya (2019); du Plessis \& Marais (2017); Paletta, Alivernini, \& Manganelli (2017); Saaduddin, Gistituati, Kiram, Jama, \& Khairani (2019); Sharp, Jarvis, \& McMillan (2020). Es decir, liderazgo no es la capacidad de alcanzar una posición, sino realmente influir en las demás personas, estén a su cargo o no. De manera similar, el liderazgo pedagógico puede abordarse de la siguiente manera: "entendemos por "liderazgo" la capacidad de ejercer influencia sobre otras personas, no basada en el poder o autoridad formal. Cuando esta influencia va dirigida a la mejora de los aprendizajes, hablamos de liderazgo pedagógico" (Bolívar, 2010b, p.34).

En el escenario escolar, el liderazgo se convierte en un aspecto estratégico en la eficiencia y calidad educativa, hasta tal punto de ser considerado "el segundo factor intra-escolar de mayor trascendencia (Leithwood \& McKinsey, citados por UNESCO, 2014). De acuerdo con Unesco (2014), la conciencia de esta importancia se ha visto en América Latina en los últimos años, al incluir entre las políticas educativas la promoción del liderazgo directivo escolar. Dicha relevancia del concepto justifica su revisión, sobre todo en lo que concierne al impacto que tiene el liderazgo en el contexto escolar y la eficacia de la institución educativa. 
Entender la forma como el liderazgo escolar o pedagógico se ha conceptualizado y los modelos causales que explican su correlación con los resultados de aprendizaje se convierten en el objetivo del presente artículo. Bajo una metodología de revisión documental se exponen, en primer lugar, los fundamentos teóricos del liderazgo escolar, haciendo énfasis en la evolución de los modelos de liderazgo y el impacto que este tiene sobre el aprendizaje estudiantil; en segundo lugar, se resaltan los resultados de algunas investigaciones empíricas que sustentan la magnitud y tipo de efecto entre estas variables; finalmente, se exponen las conclusiones relevantes halladas tras la revisión documental, como se evidencia en la literatura los artículos de Bhattacharyya (2019); du Plessis \& Marais (2017); Elswick, Cuellar, \& Mason (2019); Myende, Ncwane, \& Bhengu (2020).

\section{Fundamentos teóricos del liderazgo escolar}

El liderazgo escolar es un fenómeno que no lleva más de 100 años de ser estudiado y conceptualizado. La importancia de su investigación radica en que es una de las principales variables que inciden en un mejor desempeño de los establecimientos y del sistema general (OCDE, 2008).

No obstante, es importante resaltar que el entendimiento del liderazgo escolar no siempre ha sido desde el punto de vista educativo, pues los resultados en la práctica indican que no todo "líder" ni todo "estilo de liderazgo" son efectivos para determinados ambientes. Rodríguez (2011) realiza un análisis donde resalta la importancia, funciones y características del liderazgo pedagógico, destacándolo sobre el liderazgo centrado en la administración, como también se ve en las investigaciones de Bhattacharyya (2019); Paletta, Alivernini, \& Manganelli (2017); YanLi \& Hassan (2018). La diferencia radica en que el primero se enfoca en el currículo, enseñanza y aprendizaje, mientras que el segundo se enfoca en los sistemas de gestión administrativa, de gestión documental, cumplir la normatividad nacional, entre otras labores administrativas. Por esto es que estos líderes funcionales, orientados en lo pedagógico, tienen su impacto real y efectivo tanto en el cuerpo de profesores como en los alumnos y alumnas de los centros educativos (Rodríguez, 2011).

A continuación, se reseña la evolución de los modelos por medio de los cuales se ha estudiado y caracterizado el liderazgo escolar y los planteamientos teóricos de la influencia de este en los resultados de aprendizaje de los estudiantes.

\section{Evolución de los modelos de liderazgo escolar}

Murillo (2006) caracteriza y expone los cambios que ha tenido el liderazgo escolar a lo largo del tiempo, pasando de modelos burocráticos y centralizados hacia modelos funcionales, de liderazgo compartido y descentralizado. La figura 1 puede resumir lo expuesto por el autor en cuanto a la evolución de los enfoques. En un principio el estudio del 
liderazgo y la dirección se hacía desde un enfoque general, sin enfatizar en la escuela, encontrando la teoría de los rasgos, teoría conductual o las teorías de la contingencia. Todas ellas fundamentadas en encontrar el estilo, conducta, características, comportamientos y ambiente ideal en el cual existe un buen liderazgo.

Por un lado, la teoría de los rasgos no pudo encontrar aquellas características de personalidad, físicas o intelectuales que definían a un líder exitoso, hallando que depende del contexto en el cual de desarrolla el líder. De ahí que las teorías conductuales ahora buscarán no las características "innatas", sino los comportamientos, enfocándose en determinar cuáles eran las características del trabajo y de las conductas de los directivos. En este entorno investigativo se determinan estilos de liderazgo como el autoritario, democrático o de laissez faire; o características de la conducta efectiva de un líder como: fomento de relaciones positivas, mantener sentimientos de lealtad, conseguir altos estándares de rendimiento, tener conocimientos técnicos y coordinar y planificar

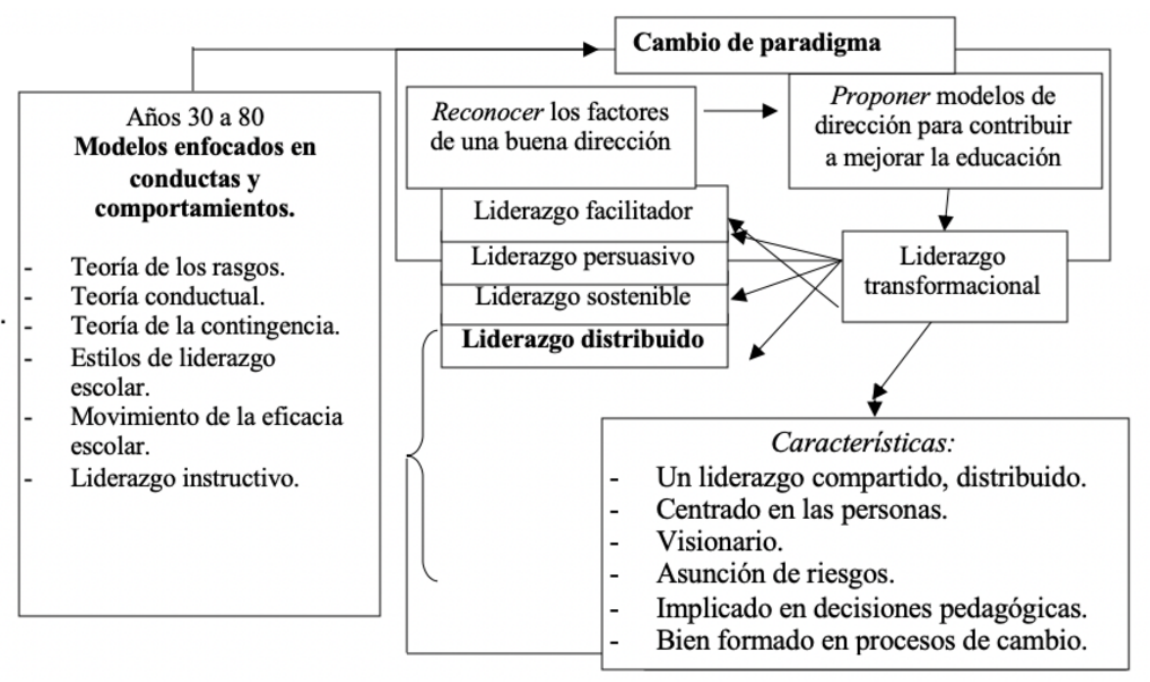

Figura 1.

Evolución del estudio del liderazgo en la escuela. Elaboración propia a partir de Murillo (2006).

No obstante, dichas características y estilos encontrados no fueron aplicables en todos los ambientes, llegando a concluir que el liderazgo depende del entorno en el cual se desarrolla. Esta condición origina el planteamiento de la teoría de la contingencia en donde el estilo de liderazgo adecuado depende de factores ambientales y relacionales donde se desarrolle. Dentro de estas teorías contingentes sobresalen factores de definen el liderazgo: relación líder-miembros, estructura de la tarea y poder de posición (Fiedler, citado por Murillo, 2006); las características de los seguidores y las demandas ambientales (House, citado por Murillo, 2006); y estilo directivo y la disposición de los seguidores (madurez) (Hersey \& Blanchard, citados por Murillo, 2006).

Una vez se adentran las investigaciones en el liderazgo escolar, en los años 60 y 70, surgen enfoques que clasifican los estilos de liderazgo en la 
escuela: técnico, humanista, educativo, simbólico, cultural, o enfocados en relaciones interpersonales, rendimiento de los alumnos, eficacia de los programas y hacia lo administrativo.

Otra tendencia en el estudio del liderazgo educativo lo componen los enfoques de la eficacia escolar para conseguir escuelas de calidad. Uno de los más importantes mencionados por Murillo (2006) es el liderazgo instructivo, el cual, a diferencia del enfoque tradicional, burocrático y centrado en la organización, se preocupa más por la enseñanza. Sin embargo, también se centra en describir las características “ideales" de una escuela que busca el desarrollo del aprendizaje, mas no de las que tenían que mejorar.

Surge entonces el enfoque del liderazgo transformacional, el cual se ubica dentro de los modelos originarios de los enfoques que buscan mejorar la educación. Por lo tanto, "este tipo de liderazgo... parte de la contribución de los directivos escolares al logro de objetivos vinculados al cambio cultural y a la resolución de problemas organizativos" (Murillo, 2006 p.43). La aplicación de este a la escuela abarca: "habilidad del director para fomentar el funcionamiento colegiado, el desarrollo de metas explícitas, compartidas, moderadamente desafiantes y factibles; $y$ la creación de una zona de desarrollo próximo para el directivo y su personal” (García-Garduño, 2010).

En los últimos años, según Murillo (2006), se ha evidenciado la aparición de enfoques que añaden ciertos elementos:

a. Liderazgo facilitador describe aquel estilo que ejerce el poder a través de los demás y no sobre ellos.

b. Liderazgo persuasivo, que se basa en que el líder crea el ambiente de optimismo, respeto, confianza e intencionalidad mediante los cuales este sugiere e incita a los cambios y consecución de metas.

c. Liderazgo sostenible, basado en el largo plazo tanto del aprendizaje, el liderazgo de otros, la justicia como del impacto en el entorno.

Bolívar (2010, p.32) explica que, de la proliferación de propuestas de modelos de liderazgo "(estratégico, sostenible, sirviente, emocional, ético, transaccional, etc.), los dos más "potentes" han sido el liderazgo instructivo o pedagógico proveniente del movimiento de escuelas eficaces y el liderazgo transformativo, vinculado al movimiento de reestructuración escolar". No obstante, una idea muy llamativa que parece que puede perdurar en el tiempo, según Murillo (2006), es el liderazgo distribuido, el cual, más allá de lo que han hecho los demás enfoques, aprovecha las habilidades de los otros en una causa común y se centra más en el liderazgo de toda la comunidad educativa que en la posición más alta en donde se encuentra el director. De ahí que el papel del director pasa de ser un gestor burocrático a un agente de cambio que sabe aprovechar las competencias de los miembros de la comunidad y encauzarlos hacia una misión en común. Este liderazgo genera un incremento de la capacidad de la escuela para resolver sus problemas, exige un papel más profesional por parte del profesorado al asumir su propio liderazgo en sus respectivas áreas y ámbitos, e implica el aprovechamiento de los conocimientos, aptitudes, destrezas, esfuerzo y la ilusión de la comunidad escolar. 


\section{Planteamientos teóricos acerca del impacto del liderazgo escolar en el aprendizaje}

Es claro que existe un impacto positivo entre un buen liderazgo escolar y los resultados de aprendizaje de los alumnos. Lo que en algunos casos no resulta tan claro son las vías causales por las que se da dicho efecto. Bolívar (2010a) expone los tipos de modelos causales que Hallinger \& Heck $(1998,2010)$ han hallado en sus investigaciones:

a. Modelo de efectos directos: son aquellos en donde el rendimiento de los alumnos depende directamente del liderazgo, así se incluyan más variables. Este modelo obedece a las primeras conceptualizaciones del liderazgo en donde la escuela depende en gran medida de la acción del líder. Los efectos directos, de acuerdo con Dhuey \& Smith (2014), surgen cuando los directores interactúan de manera directa con los estudiantes mediante el monitoreo, la sanción de la conducta, control de disciplina, la evaluación y mejora de la educación de sus alumnos.

b. Modelo de efectos mediados: en este enfoque el liderazgo tiene el papel de crear un contexto. Es decir, el liderazgo primero pasa por las prácticas docentes, la cultura escolar, el desarrollo profesional, etc., para ahí sí impactar el aprendizaje estudiantil. Se da un fenómeno que se puede denominar "efecto derrame", donde las capacidades y aprendizaje de los profesores desborden finalmente hacia sus alumnos.

c. Modelo de efectos inversos: corresponde a una visión en la que los resultados de la escuela generan un ímpetu para el desarrollo del liderazgo.

d. Modelo de efectos recíprocos: consiste en la relación mutua entre las propias variables del liderazgo y no solo entre el liderazgo y las variables mediadoras. Las variables liderazgo, capacidad de mejora y aprendizaje de los estudiantes se influencian mutuamente a lo largo del tiempo.

De acuerdo con Bolívar (2010a), los estudios encontrados en el primer modelo (efectos directos), resultan insuficientes, mientras que existe mayor evidencia del segundo modelo (efectos mediados). Respecto al tercer modelo (efectos inversos), no hay evidencia empírica, pero los investigadores reconocen este tipo de relación, mientras que, el cuarto modelo (efectos recíprocos), es uno de los más extendidos.

Un ejemplo de este cuarto modelo es la conceptualización de las variables que influyen en el aprendizaje de los estudiantes hecha por Day et al. (2009), en la cual la influencia del liderazgo escolar (variable independiente), en el aprendizaje de los estudiantes (variable dependiente), se ve condicionada por variables moderadoras y mediadoras, las cuales también se influyen mutuamente (ver figura 2). Las primeras consisten en características de los docentes y los estudiantes que condicionan el efecto del liderazgo, mientras que las segundas consisten en características de la escuela sobre las cuales el liderazgo tiene un efecto directo. 


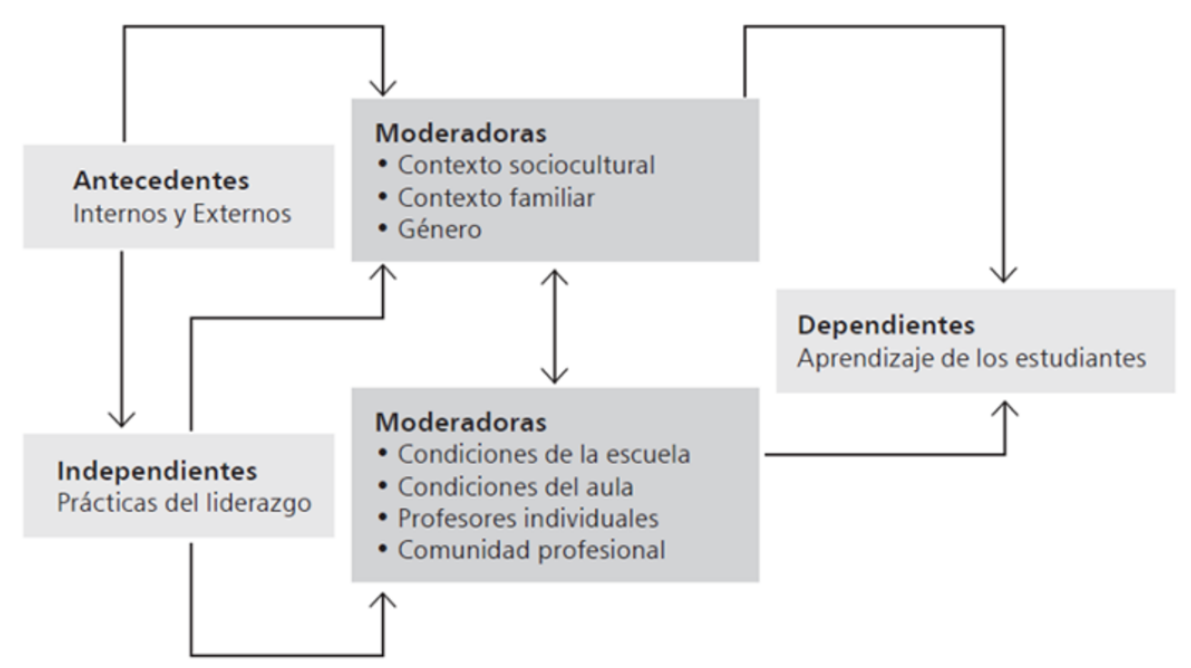

Figura 2.

Variables intervinientes en el aprendizaje de los estudiantes Fuente: reelaboración de Bolívar (2010a), basado en Hallinger \& Heck $(1998,2010)$.

Bolívar (2010) coincide con estos planteamientos al identificar la escuela como el lugar donde la dirección no se sitúa en la cumbre de la pirámide, sino mediando una red de relaciones (ver figura 3). En esta red de relaciones, el liderazgo contribuye a incrementar el aprendizaje de los alumnos indirectamente, por medio de su influencia en el profesorado o en otros aspectos de la organización (Bolívar, 2010b). Esta última relación puede evidenciarse cuantitativamente en la figura 4, en donde Bolívar (2010b) indica el nivel de influencia que existe en la relación causal de las variables. En este caso, el director puede influir en gran medida sobre las condiciones de trabajo, sin embargo, estas tienen una influencia baja en el cambio de prácticas y en el rendimiento escolar; así mismo, la influencia del director en las capacidades docentes es baja, no obstante, la influencia de estas sobre el rendimiento escolar es alta. 


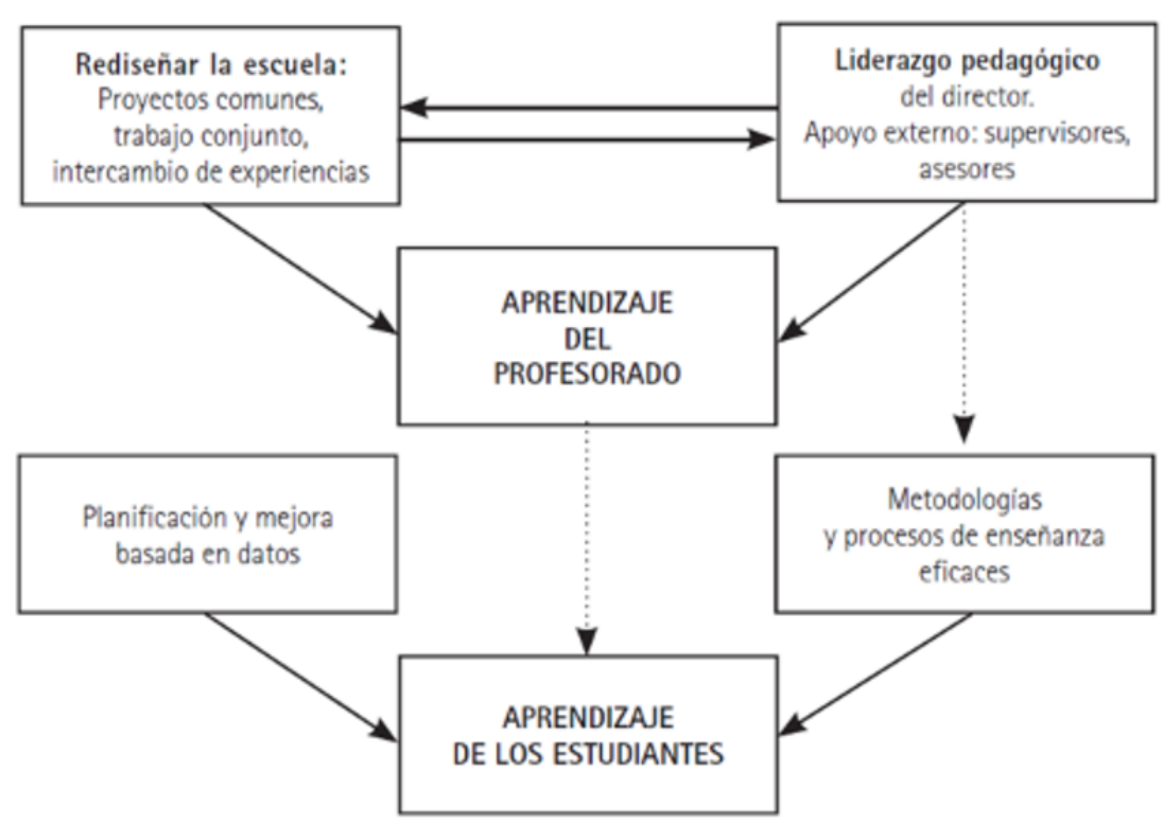

Figura 3.

La escuela como organización para el aprendizaje. Fuente: Bolívar (2010b).

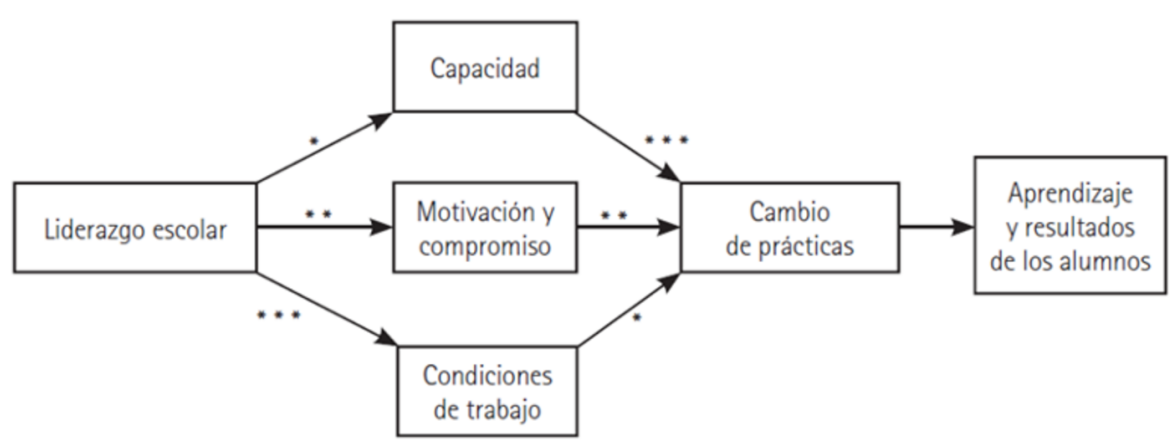

$\because$ : baja influencia, ${ }^{*}$ : moderada, ${ }^{\cdots *}$ : alta

Figura 4.

Efectos del liderazgo escolar.

Fuente: Bolívar (2010b).

Por su parte, Leithwood (2011) conceptualiza la influencia del liderazgo sobre el aprendizaje de los alumnos, argumentando que en gran medida es indirecta. Esta influencia indirecta se lleva a cabo por medio de cuatro sendas: racional (calidad de la enseñanza, retención de los docentes, eficacia propia y colectiva de los docentes, currículo, presión académica, entre otros); emocional (eficacia docente, compromiso, estrés, confianza, ánimo); organizacional (tiempo pedagógico, complejidad de la carga de trabajo docente, redes profesionales, estructuras que apoyen la colaboración), y familiar (expectativas de los padres, modelos parentales, conexiones con los adultos, espacio y tiempo en el hogar para trabajo relacionado con la escuela). 


\section{MÉTODO}

La presente investigación se basa en una revisión de literatura, por tanto, se realiza esta metodología mediante la revisión de algunos documentos que indican la tendencia sobre este fenómeno.

\section{Investigación empirica acerca de los efectos del liderazgo escolar}

Existe abundancia de estudios que han medido con diferentes metodologías el efecto del liderazgo en el aprendizaje de los estudiantes en el entorno escolar. La mayoría de los primeros estudios se han realizado en habla inglesa, y en los últimos años se han implementado a nivel de Latinoamérica. El tema ha sido trabajado a tal punto que existen estudios de revisión documental que realizan meta-análisis para obtener conclusiones.

Por ejemplo, Sun \& Leithwood (2014), tras una revisión documental de 24 estudios que evaluaban efectos directos del Liderazgo Transformacional Escolar (LTE) (Kvashnina \& Martynko, 2016), en el logro de los estudiantes, encuentran una relación pequeña, pero con significativo efecto positivo directo, con un coeficiente de correlación promedio de 0.09. Los autores también encuentran que algunas prácticas de LTE como "construir estructuras de colaboración" y "proporcionar consideración individualizada" hicieron contribuciones mucho más grandes en los logros de los estudiantes $(r=0.17$ y $r=0.15$ respectivamente), que otras prácticas, las cuales oscilaron entre $r=0.03$ y $\mathrm{r}=0.05$. "El uso de métodos de liderazgo por parte de los docentes con respuestas positivas, discursos inspiradores y elogios pueden generar resultados y la interacción con los pares, mientras que enseñar el comportamiento establece un estilo moral para generar una sensación de logro" (Lan, Chang, Ma, Zhang, \& Chuang, 2019, p.53).

En cuanto a efectos indirectos por medio de variables mediadoras y moderadoras, Sun \& Leithwood (2014) encuentran resultados mixtos, pues para algunas investigaciones como la de Solomon (2007), informó una elevada correlación $(\mathrm{r}=0.79)$ entre el LTE, el compromiso del profesorado, la eficacia colectiva, el SES y el rendimiento de los estudiantes; mientras que Nicholson (2003), no encontró una asociación significativa entre el LTE y la eficacia colectiva del profesorado; y Sun (2010), en un análisis más detallado, encontró que los efectos del LTE sobre el logro de los estudiantes no son moderados por el nivel escolar (primaria, media, secundaria).

El liderazgo transformacional también obtuvo una "pequeña" influencia positiva sobre los resultados de los estudiantes en la revisión documental realizada por Robinson, Lloyd, \& Rowe (2014), quienes utilizaron una medida de la magnitud del efecto de la desviación estándar, encontrando que el liderazgo instructivo $(\mathrm{ES}=0,42)$ tiene un impacto tres a cuatro veces mayor que el transformacional $(E S=0,11)$ y otros tipos de liderazgo $(E S=0,30)$. Este resultado se debe a que el liderazgo 
transformacional se centra más en la relación entre líderes y docentes y la calidad de sus relaciones, que en la relación con los estudiantes.

Para el caso de esta revisión también se analiza, dentro del contexto colombiano y del desarrollo de América Latina que les permita aportar una nueva generación de líderes empresariales, emprendedores e innovadores que apoyen la transformación productiva, la creación de nuevas empresas para el desarrollo sostenible del país y de sus zonas menos productivas, que hoy tienen una oportunidad de convertirse en locomotoras de desarrollo, con empresas que creen valor a su comunidad (Arias-Velandia et al., 2018).

De manera similar, Horn (2013) encuentra que el liderazgo escolar tiene mayor efecto en los resultados de los profesores que de los estudiantes, pues liderazgo explica un $7 \%$ de la varianza del rendimiento de los estudiantes en el caso de lenguaje y el $8 \%$ de la varianza en el caso de matemáticas, mientras que estos efectos son mayores en los detectados entre el liderazgo escolar y el desempeño docente (que explican entre el $11 \%$ y el $28 \%)$.

Por su parte, un estudio que mide los efectos directos entre liderazgo y aprendizaje en la escuela es el de Freire \& Miranda (2014). Con los datos de la Encuesta Escolar en el marco del estudio Niños del Milenio, el cual se realiza en cuatro países en vías de desarrollo: Etiopia, India, Perú y Vietnam, encuentran que la correlación entre liderazgo pedagógico y rendimiento académico de los estudiantes es positiva y significativa; en el caso de la comprensión lectora fue de $\mathrm{r}=0.25$ y en matemáticas fue de $r=0.14$. No obstante, los autores entienden el condicionamiento de variables mediadoras y moderadoras, pues descubren que el liderazgo del director puede tener mejor impacto en el rendimiento de los estudiantes si las condiciones de la escuela y las practicas pedagógicas son las adecuadas, es decir, el nivel de preparación y conocimiento sobre cómo enseñar los contenidos cumplen un papel importante como condiciones previas para que dicho liderazgo tenga efectos sobre la eficacia de la enseñanza. Finalmente, el estudio también encuentra que el liderazgo pedagógico afecta positivamente en mayor medida a los estudiantes de bajo y promedio rendimiento, los cuales se ubican generalmente en escuelas rurales y contextos de bajo nivel socioeconómico.

Otros estudios han evaluado los resultados del liderazgo no solo en función del aprendizaje en los estudiantes. Ruiz (2011) encontró que la correlación de los estilos de liderazgo transformacional y transaccional en la eficacia de las instituciones educativas fue de $\mathrm{r}=0.57 \mathrm{y} \mathrm{r}=0.56$, respectivamente. En este caso, la eficacia de la institución no solo tuvo en cuenta los logros a nivel de los aprendizajes sino a nivel de capacidades docentes, nivel curricular, logro de los propósitos del Proyecto Educativo Institucional, compromiso de los docentes y logro en los equipos docentes.

En cuanto al modelo de liderazgo que ha tenido mayor acogida en los últimos años, el liderazgo distribuido, López \& Gallegos (2017) confirman que este presenta incidencia estadísticamente significativa en los resultados de aprendizajes en la prueba SIMCE matemática en los 
establecimientos educativos en estudio. A conclusiones similares llegan Hallinger \& Heck en Harris (2009), quienes confirman que, tras resumir los resultados de varios estudios, el liderazgo distribuido es un co-efecto importante de los procesos de mejoramiento escolar.

Por su parte, Bolívar (2010a) reelabora los resultados obtenidos por Leithwood, Harris, \& Hopkins (2008), acerca de los resultados del liderazgo distribuido en el profesorado y el alumnado. Como se puede observar en la figura 5, el grado de correlación difiere del marco conceptual propuesto por Bolívar (2010b) en la figura 4. En este caso, la mayor influencia del liderazgo en las variables mediadoras (indirectas) se halla hacia las condiciones de trabajo de los profesores $(\mathrm{r}=0.55)$, no obstante, es la variable intermedia que menos impacta los logros académicos y los resultados $(\mathrm{r}=0.08)$; mientras que con la variable "motivación y compromiso" la correlación es la más baja $(\mathrm{r}=0.25)$, pero el efecto en los logros académicos es más alta $(\mathrm{r}=0.65)$. Si hallamos la totalidad del efecto del liderazgo en los logros académicos se puede determinar que, vía condiciones de trabajo la correlación es de $\mathrm{r}=0.044\left(0.55^{*} 0.08\right)$, vía motivación y compromiso es de $\mathrm{r}=0.1625$ y vía capacidad es $\mathrm{r}=0.1748$. El mayor impacto del liderazgo distribuido en los logros académicos de los estudiantes se logra mejorando la capacidad de los profesores. Finalmente, la suma total de estos tres efectos permite concluir que el liderazgo escolar distribuido, según este estudio, impacta con un índice de correlación de 0.3813 los logros académicos de los estudiantes.

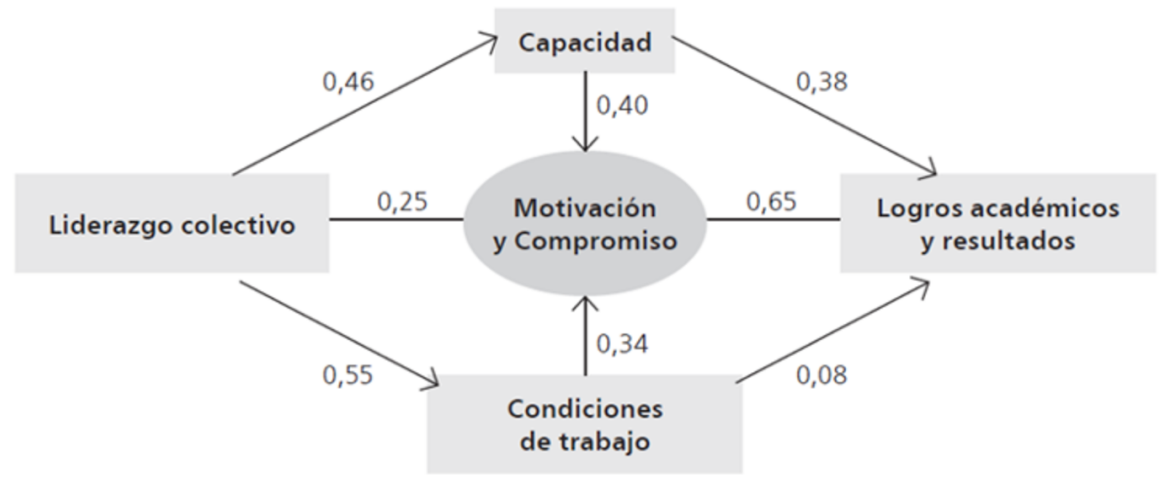

Figura 5.

Efectos del liderazgo distribuido en profesorado y alumnado. Fuente: Bolívar (2010a), adaptado de Leithwood et al. (2008).

Es importante resaltar que estos resultados hallados por Leithwood et al., (2008), también reconocen efectos mutuos entre variables mediadoras (de $\mathrm{r}=0.40 \mathrm{y} \mathrm{r}=0.34$ ), lo cual concuerda con el cuarto modelo de relaciones causales recíprocas de Hallinger \& Heck $(1998,2010)$.

Finalmente, uno de los estudios más completos y que se ubica también dentro del modelo de efectos recíprocos fue realizado por Day et al. (2009), quienes buscaron crear un modelo (ver figura 6), que involucrara las dimensiones clave del liderazgo (color rojo y rojo claro), las dimensiones de la práctica del liderazgo distribuido (color naranja), las dimensiones que funcionan como factores mediadores (color azul), y las dimensiones de los resultados intermedios (color verde), que 
tienen efectos directos o indirectos sobre los cambios en los resultados académicos (Wills, 2016) de los alumnos (color amarillo), a lo largo de tres años. De izquierda a derecha se evidencian las correlaciones entre las dimensiones de liderazgo y las dimensiones mediadoras, entre estas y los resultados intermedios y entre estos últimos y los resultados académicos.

Al final, bajo este modelo, son tres las variables que influyen en rendimiento académico de los estudiantes: personal $(\mathrm{r}=0.12)$, aprendizaje y enseñanza $(\mathrm{r}=0.11)$ y cambio en el comportamiento de los alumnos $(\mathrm{r}=0.14)$. Este complejo entramado de relaciones evidencia la dificultad de establecer una dirección directa inequívoca entre liderazgo y resultados en el aprendizaje de los alumnos.

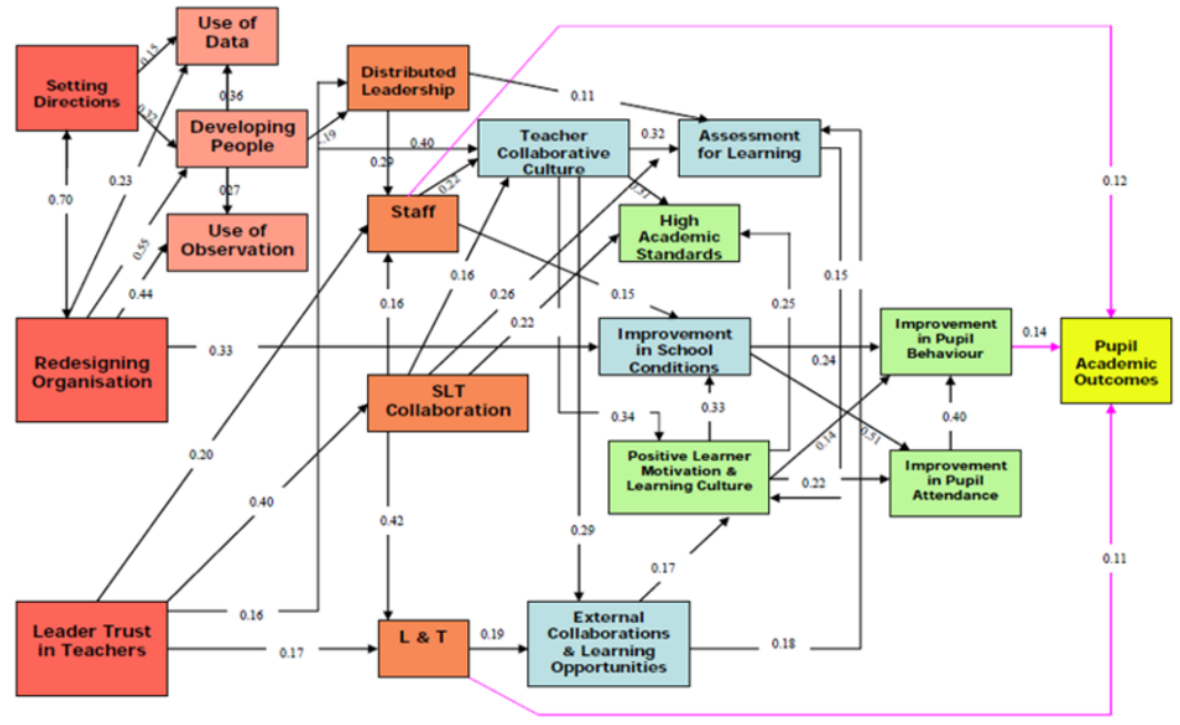

Figura 6.

Modelado de Ecuaciones Estructurales basado en la percepción de las principales prácticas de liderazgo y el cambio en los resultados de los alumnos durante tres años (2003-2005). Fuente: Day et al. (2009).

\section{DISCUSIÓN}

El liderazgo escolar se constituye en una de las variables de mayor influencia en la eficacia de las instituciones educativas. Su entendimiento no siempre fue tan flexible y detallado, pues ha ido evolucionando de concepciones rígidas, burocráticas y administrativas a modelos mucho más transformacionales, distribuidos y pedagógicos.

Por su parte, los planteamientos teóricos han caracterizado los efectos del liderazgo en el aprendizaje mediante modelos que distinguen influencias directas, indirectas, inversas y recíprocas, entre variables de tipo dependiente, independientes, mediadoras y moderadoras.

Así mismo, existe evidencia empírica suficiente para demostrar que hay un impacto positivo (directo e indirecto), entre liderazgo escolar y aprendizaje. No obstante, los efectos directos son de baja o nula correlación. En su lugar, existe un "efecto derrame" en el que el liderazgo influye primero variables mediadoras, y de ahí influencia los resultados 
del aprendizaje. Esto se verifica en el hecho de que existen índices de correlación mucho más altos entre dimensiones de liderazgo y variables intermedias como motivación y capacidades de los docentes, que entre las dimensiones del liderazgo y el rendimiento de los estudiantes.

Es necesario tener en cuenta que los estudios que deseen realizarse acerca del impacto de liderazgo escolar en los resultados académicos de los estudiantes deben tener muy claro el enfoque teórico bajo el cual se construye y las herramientas metodológicas a usarse, pues, como pudo observarse, son diversas las variables directas e indirectas que influyen en el aprendizaje de los estudiantes. Estos puntos son de vital importancia para adelantar investigaciones en la región, en donde aún son muy escasos.

\section{Referencias}

1. Bhattacharyya, E. (2019). Leadership competencies and leadership style in aspiring cluster schools of excellence. PertanikaJournal of Social Sciences and Humanities, 27(2), 1111-1129. Recuperado de: https://www.scopus.com/inward/record.uri?eid=2-s2.0-850691614 78\&partnerID $=40 \& \mathrm{md} 5=739763111 \mathrm{~cd} 3151 \mathrm{ff9f56b46af94b5b7}$

2. Bolívar, A. (2010a). ¿Cómo un liderazgo pedagógico y distribuido mejora los logros académicos? Revisión de la investigación y propuesta. Revista Internacional de Investigación en Educación, 3(5), 79-106.

3. Bolívar, A. (2010b). Liderazgo para el aprendizaje. Organización y Gestión Educativa, (1), 15-20.

4. Day, C., Sammons, P., Hopkins, D., Harris, A., Leithwood, K., Gu, Q., ... Kington, A. (2009). The Impact of School Leadership on Pupil Outcomes: Final Report (Investigación No. DCSF-RR108). University of Nottingham.

5. du Plessis, E., \& Marais, P. (2017). A Grounded Theory Perspective on Leadership in Multicultural Schools. Journal of Asian and African Studies, 52(5), 722-737. https://doi.org/10.1177/0021909615612122

6. Freire, S., \& Miranda, A. (2014). El rol del director en la escuela: el liderazgo pedagógico y su incidencia sobre el rendimiento académico. Avances de investigación, 17, 1-64.

7. Escuelas primarias públicas de México. Los primeros años en el puesto. Revista Iberoamericana sobre Calidad, Eficacia y Cambio en Educación, 8(4), 2735. https://doi.org/10.1104/pp.110.164053

8. Elswick, S., Cuellar, M., \& Mason, S. (2019). Leadership and School Social Work in the USA: A Qualitative Assessment. School Mental Health, 11 (3), 535-548. https://doi.org/10.1007/s12310-018-9298-8

9. Hallinger, P., \& Heck, R. (1998). Exploring the principal contribution to school effectiveness: 1980- 1995. School Effectiveness and School Improvement, 9(2), 157-191.

10. Hallinger, P., \& Heck, R. (2010). Collaborative leadership and school improvement: understanding the impact on school capacity and student learning. School Leadership \& Management, 30(2), 95-110.

11. Kvashnina, O., \& Martynko, E. (2016). Analyzing the potential of flipped classroom in ESL teaching. International Journal of Emerging Technologies in Learning, 11(3), 71-73. https://doi.org/10.3991/ijet.v11i03.5309 
12. Harris, A. (Ed.). (2009). Distributed Leadership: Different Perspectives. London: Springer Science \& Business Media.

13. Horn, A. (2013). Liderazgo escolar en Chile y su influencia en los resultados de aprendizaje (Tesis Doctoral). Universidad Autónoma de Madrid.

14. Leithwood, K., Harris, A., \& Hopkins, D. (2008). Seven strong claims about successful school leadership. School Leadership and Management, 28(1), 27-42.

15. López, P., \& Gallegos, V. (2017). Liderazgo distribuido y aprendizaje de la matemática en escuelas primarias: el caso de Chile. Perfiles educativos, 39(158), 112-129.

16. Murillo, J. (2006). Una dirección escolar para el cambio: del liderazgo transformacional al liderazgo distribuido. Revista Iberoamericana sobre Calidad, Eficacia y Cambio en Educación, 4(4e), 11-24.

17. Myende, P., Ncwane, S., \& Bhengu, T. (2020). Leadership for learning at district level: Lessons from circuit managers working in deprived school contexts. Educational Management Administration and Leadership. https ://doi.org/10.1177/1741143220933905

18. Nicholson, J. (2003). An exploration of the ability to predict student achievement from leadership behaviors, teacher job satisfaction, and socioeconomic status. Abstracts International, 64(03).

19. Arias-Velandia, N., Rincón-Báez, W., Becerra-Plaza, G., Mejía-Rodríguez, A., Salas-Díaz, R. (2018). Retos a los programas de administración, una mirada al saber. Resultados de la evaluación externa (2012-2016). Bogotá: Institución Universitaria Politécnico Grancolombiano.

20. OCDE. (2008). Education and training policy. Improving school leadership: Policy and practice. OCDE.

21. Paletta, A., Alivernini, F., \& Manganelli, S. (2017). Leadership for learning: The relationships between school context, principal leadership and mediating variables. International Journal of Educational Management, 31(2), 98-117. https://doi.org/10.1108/IJEM-11-2015-0152

22. Robinson, V., Lloyd, C., \& Rowe, K. (2014). El impacto del liderazgo en los resultados de los estudiantes. Un análisis de los efectos diferenciales de los tipos de liderazgo. Revista Iberoamericana sobre Calidad, Eficacia y Cambio en Educación, 12(4), 13-40.

23. Rodríguez, G. (2011). Funciones y rasgos del liderazgo pedagógico en los centros de enseñanza. Educación y educadores, 253-267.

24. Ruiz, G. (2011). Influencia del estilo de liderazgo del director en la eficacia de las instituciones educativas del consorcio «Santo Domingo de Guzmán» de Lima Norte (Tesis de maestría). Universidad Nacional Mayor de San Marcos, Lima.

25. Saaduddin, S., Gistituati, N., Kiram, P., Jama, J., \& Khairani, Y. (2019). The effects of principal leadership on effective school management. International Journal of Innovation, Creativity and Change, 5(6), 359-367. Recuperado de: https://www.scopus.com/inward/record.uri?eid=2-s2.0-850844795 15\&partnerID=40\&md5=a942aad510a1ea42fdaf75d9f5769522

26. Sharp, K., Jarvis, J., \& McMillan, J. (2020). Leadership for differentiated instruction: teachers' engagement with on-site professional learning at an Australian secondary school. International Journal of Inclusive Education, 24(8), 901-920. https://doi.org/10.1080/13603116.2018.1492639 
27. Solomon, C. (2007). The relationships among middle level leadership, teacher commitment, teacher collective efficacy and student achievement (Tesis Doctoral).

28. Sun, J. (2010). A review of transformational leadership research: A meta-analytic approach (Tesis doctoral inédita). University of Toronto, Toronto.

29. Sun, J., \& Leithwood, K. (2014). Efectos del liderazgo escolar transformacional en el rendimiento de los estudiantes. Revista Iberoamericana sobre Calidad, Eficacia y Cambio en Educación, 12(4), 41-70.

30. UNESCO. (2014). El liderazgo escolar en América Latina y el Caribe: un estado del arte con base en ocho sistemas escolares de la región. Imbunche Ediciones Ltda.

31. Yan-Li, S., \& Hassan, D. (2018). Leadership behaviour on job satisfaction in Malaysian national secondary schools: Motivation and hygiene satisfaction. Malaysian Online Journal of Educational Management, 6(3), 48-67. https://doi.org/10.22452/mojem.vol6no3.3

32. Wills, G. (2016). Principal leadership changes and their consequences for school performance in South Africa. International Journal of Educational Development, 51, 108-124. https://doi.org/10.1016/j.ijedudev.2016.08. 005 